\title{
Psoríase e sua relação com aspectos psicológicos, stress e eventos da vida
}

\author{
Psoriasis and its relation with psychological \\ aspects, stress, and life events
}

\author{
Kênia de Sousa SILVA \\ Eliana Aparecida Torrezan da SILVA²
}

\begin{abstract}
Resumo
Foi realizado um levantamento na literatura científica quanto à relação entre psoríase, aspectos psicológicos, stress e eventos de vida. Foi verificado que os pesquisadores da área concordam que a psoríase tem etiologia desconhecida. No entanto comentam que existem fatores genéticos, endógenos e ambientais relacionados ao aparecimento e à piora da doença. A literatura mostra que os aspectos psicológicos e o stress podem colaborar para o surgimento, recidiva ou piora do quadro clínico. A estigmatização em relação à aparência física da pessoa com psoríase é vista como um fator estressante. Os dermatologistas e psicólogos concordam quando o assunto é o tratamento da psoríase, salientando que, além da intervenção clínica medicamentosa, é recomendável a psicoterapia cognitivo-comportamental como estratégia para a melhora ou controle da doença. Pesquisas na área são sugeridas para estabelecer correlações entre as variáveis investigadas no presente artigo.
\end{abstract}

Unitermos: aspectos psicológicos; eventos de vida; psoríase; stress.

\begin{abstract}
The present study had the objective to research in the Scientific Literature the relation among psoriasis, psychological aspects, events of life as well as stress. It was verified that the scientists agree psoriasis has unknown etiology. However, they comment the existence of genetic endogenous and ambient factors related to the appearance and worsening of the illness. Scientists stand out the psychological aspects, emotional state and stress can collaborate to the illness return or worsening. The stigmatization in relation to physical appearance can be a stress source for the patients. The dermatologists and psychologists agree on the treatment of psoriasis. They point out that, beyond the clinical treatment, the psychotherapy behavioral cognitive is recommendable as an intervention strategy for improvement and/or control of the illness. Researches in the area are suggested in order to establish correlation among the variables investigated in the present article.
\end{abstract}

Uniterms: psychological aspects; events of life; psoriasis; stress.

As células que originam a pele têm uma ligação muito próxima com as células nervosas. Observa-se que muitas doenças da pele têm alguma relação com o estado emocional da pessoa. As alterações emocionais podem se manifestar por algum órgão do corpo, chamado de "órgão de choque". A pele é um desses "órgãos de choque" e, certamente, sofre em função dessas oscilações emocionais (Steiner \& Perfeito, 2003).

$\boldsymbol{\nabla} \boldsymbol{\nabla} \boldsymbol{\nabla}$

1 Universidade Metodista de Piracicaba, Departamento de Administração. Rod. Do Açúcar, km156, Campus Taquaral, Bloco 7, Caixa Postal 68, 13400-911, Piracicaba, SP, Brasil. Correspondência para/Correspondence to: K.S. SILVA. E-mail: <kenia@laal..com.br>.

2 Universidade Camilo Castelo Branco, Faculdade de Psicologia. São Paulo, SP, Brasil. 
Segundo especialistas da área, uma das formas do indivíduo expressar insatisfação, mal-estar ou desconforto é por meio da somatização, ou seja, através da liberação de maneira não intencional dessa emoção em um ou mais órgãos do corpo.

Estudiosos tentam explicar esse processo de somatização. McDougall (1996) menciona que o paciente que somatiza não se caracteriza pela incapacidade de vivenciar ou exprimir as emoções e sim pela incapacidade de suportar a contenção do excesso e da experiência afetiva.

Steiner e Perfeito (2003) comentam algumas dermatoses e suas relações com a área psicológica. A dermatite atópica ocorre normalmente associada à asma, à rinite alérgica ou à urticária. É uma doença crônica, com períodos de crise e acalmia, e tem relação com o stress, que pode desencadear uma crise. A dermatite seborréica é uma afecção crônica recorrente: observa-se a participação de fatores emocionais nas recidivas e no agravamento do quadro. O líquen simples crônico ou neurodermite consiste de uma placa liquenificada extremamente pruriginosa e de evolução crônica; ocorre uma resposta cutânea a um estímulo inicial, sendo, entretanto, o fator emocional o mais importante, observando-se sempre, como base, a ansiedade ou a compulsão.

A acne vulgar, dermatose, é extremamente comum entre os adolescentes e pode ser representada por quadros de intensidade variável, que vão desde comedões, pápulas, até pústulas, cistos e trajetos fistulosos que sofrem influência do stress, confirmada pela maioria dos pacientes com acne.

O vitiligo, discromia adquirida, caracterizado por máculas acrômicas, não tem sua etiologia completamente esclarecida, entretanto se observa que o stress emocional ou físico pode desencadear ou agravar o quadro. A psoríase, assim como as outras dermatoses, está associada ao stress no seu desencadeamento ou na piora das lesões. Além disso, na psoríase a própria doença está freqüentemente vinculada ao desencadeamento do stress emocional pelo constrangimento das lesões (Steiner \& Perfeito, 2003).

Rodrigues (1997) acrescenta que o paciente com psoríase tem dificuldade em expressar suas emoções,

258 principalmente as agressivas e hostis. Para Mingorance
(1999), a psoríase poderia ser uma forma não verbal de expressar os sentimentos.

Estudo realizado por Allegranti, Gon, Magaton-Rizzi e Aguglia (1994), com pacientes psicossomáticos, incluindo aqueles que sofrem de condições dermatológicas, dá atenção considerável ao predomínio de personalidades que têm um restrito estilo cognitivo-afetivo, dificuldades de expressar verbalmente suas emoções, deficiências nas habilidades introspectivas e são consideradas como personalidades alexetímicas.

Nesse estudo foi avaliada a prevalência de características de alexetimia em uma amostra diagnosticada com psoríase. Foram trinta e dois pacientes avaliados, utilizando-se instrumentos específicos para alexetima: Toronto Alexethymia Scale (TAS) e o teste de personalidade Rorschach. Os testes foram comparados com um grupo-controle. Quanto à escala de alexetimia, os pacientes tiveram mais características de alexetimia do que o grupo-controle, porém sem diferença estatística significante. Foi encontrada alta porcentagem de borderline no grupo-controle. Segundo os autores, os pacientes apresentam características secundárias de alexetimia: eles reagem afetivamente negativos nas emoções com o propósito de se protegerem dos outros.

A respeito do Rorschach, todos os pacientes têm fantasias e afetividade diminuídas em relação ao grupo-controle, pouca adaptação de afeto e um percentual de esterotipia maior. Refere ainda o estudo que os pacientes têm um estilo de comunicação simbólico diminuído e que o uso de terapia pode ajudá-los a organizarem seus sentimentos internos.

Tomando-se por base as considerações anteriormente apresentadas, o presente trabalho volta-se para o levantamento na literatura científica sobre a psoríase, aspectos psicológicos, stress e eventos de vida conforme apresentação nos parágrafos posteriores.

\section{Psoríase}

Psoríase caracteriza-se por ser uma doença de etiologia desconhecida, com evolução crônica, acentuada e tendência às recidivas. A lesão característica é representada por uma placa eritemato-escamosa, saliente em relação à superfície da pele. As escamas estão superpostas como lâminas de um fragmento de mica; apresentam cor avermelhada e podem ser 
destacadas com facilidade, mediante a raspagem da lesão. As lesões podem ser do tamanho de gotas (gutata) ou de moedas (numular); podem confluir para formação de figuras policíclicas, como também branquear o centro e permanecer em atividade na periferia, formando arcos de círculo (gyrata).

A psoríase tem elevada predileção pelo couro cabeludo e pelas superfícies de extensão de joelhos e cotovelos, mas qualquer área cutânea pode ser sede de lesões. As unhas são freqüentemente envolvidas, e traumatismos de qualquer natureza, que atingem a pele do paciente, podem produzir o aparecimento de novas lesões na área traumatizada. A generalização por toda a pele denomina-se forma entrodérmica. Formas de difícil controle são representadas pelas psoríases artropática e eritrodérmica (Proença \& Maia, 1995).

De acordo com Sampaio, Castro e Rivitti (1989), a psoríase artropática é aquela cujas lesões são disseminadas com comprometimento das articulações (particularmente das mãos), que se apresentam inchadas e dolorosas; psoríase pustulosa com um quadro de lesões eritemato-escamosas e pustuloso generalizado, ampla exacerbação de formação de pústula na pele, levando o indivíduo a apresentar febre; e, finalmente, a psoríase eritrodérmica, que é uma descamação universal ou muito extensa, podendo ter um aumento até que todo o corpo seja envolvido.

Para alguns estudiosos, o tipo mais comum de psoríase é a denominada vulgar. A psoríase vulgar é caracterizada por pápulas e placas róseas e vermelhas que são de tamanho variável, nitidamente demarcadas, secas e usualmente cobertas com camadas de finas escamas prateadas. O couro cabeludo, a região sacra e as superfícies extensoras das extremidades são comumente afetados, embora em alguns pacientes as áreas flexurais e intertriginosas (psoríase inversa) sejam principalmente afetadas (Elder et al., 2001).

Chiozza (1991) acrescenta que a psoríase vulgar evolui em episódios separados, com períodos de latência inconstantes, ao acaso e imprevisíveis, e com momentos de remissão total. O prognóstico e o tratamento dependem da gravidade e da extensão do acometimento inicial.

Segundo Berkow, Beers, Bogin e Fletcher (2003), a psoríase é uma doença comum que afeta de 2\% a 4\% dos brancos. Os negros apresentam menor tendência a apresentá-la. A psoríase se manifesta, mais freqüentemente, em indivíduos entre 10 e 40 anos de idade, embora todas as faixas etárias estejam a ela suscetíveis.

Borke Brauninger (1998) mencionam que a predisposição ao desenvolvimento da psoríase tem caráter hereditário, mas sua causa permanece desconhecida.

Brophy, Taylor, Blake e Calin (2003) concordam que há um componente genético na psoríase e estudos demonstram que se trata de uma doença auto-imune da pele. Uma pessoa pode nascer com uma predisposição genética para psoríase: esse dado foi encontrado em uma amostra de pacientes com psoríase, em que metade dos entrevistados relatou incidência de casos de diagnóstico na família.

O curso da doença varia individualmente, porém, em geral, tende a assumir um caráter crônico; muitos pacientes podem apresentar diversas formas de recidiva e piora do quadro. Para que a doença possa se manifestar, é necessária a presença de diversos fatores, cuja importância varia individualmente. Entre esses, podem ser citados irritação da pele causada por pressão, traumatismos e queimaduras por exposição ao sol, abuso crônico de álcool, stress físico, alguns medicamentos, infecções crônicas ou obesidade (Bork \& Brauninger, 1998).

Ressalta-se a importância de diagnosticar a psoríase, pois, muitas vezes, o quadro pode ser avaliado erroneamente, uma vez que outros distúrbios também podem produzir placas e descamação similares. À medida que a psoríase evolui, o padrão descamativo característico é geralmente fácil de ser reconhecido pelo médico e, por essa razão, os exames diagnósticos comumente não são necessários. Contudo, para confirmar o diagnóstico, o médico pode realizar uma biópsia de pele (Berkow et al., 2003).

Observa-se que, quanto mais cedo ocorrer a doença, pior o prognóstico. Com relação à cura, não se conhece um método terapêutico, porém é possível controlar a doença na maioria dos casos (Chiozza, 1991).

No que se refere ao tratamento médico, quando um indivíduo apresenta apenas algumas poucas placas pequenas, a psoríase responde rapidamente à intervenção, com o uso de pomadas e cremes emolientes que ajudam a manter a pele úmida. Pomadas contendo 
corticóides, vitamina D, ácido salicílico também são muito eficazes para alguns pacientes. A luz ultravioleta (PUVA) e a exposição ao sol também podem ajudar a eliminar sintomas da psoríase. No entanto o tratamento com PUVA pode aumentar o risco de câncer de pele devido à ação da luz ultravioleta e, por essa razão, o tratamento deve ser supervisionado rigorosamente por um médico (Berkow et al., 2003).

Estudo realizado por Nijsten, Margolis, Feldaman, Rolstad e Stern (2005) objetivou avaliar a satisfação dos pacientes psoriáticos com quatro opções sistemáticas de tratamento disponíveis antes de 2002. A amostra foi composta por 1197 pacientes recrutados aleatoriamente na população geral dos E.U.A., dentre os integrantes da Fundação da Psoríase e das pessoas que apenas contataram a fundação. Foi utilizada uma entrevista que incluía perguntas sobre o uso e a satisfação com as terapias específicas que tratam psoríase. A pesquisa revelou que $26 \%$ dos entrevistados indicaram uso de methotrexate, psoralen mais o ultravioleta A (PUVA), cyclosporin e/ou acetretin.

Comparando os entrevistados com indivíduos que nunca haviam usado nenhum desses tratamentos, os usuários relataram doença mais extensiva e contagens mais elevadas do índice da inabilidade da psoríase. Mais de um terço da amostra estava insatisfeita com cada terapia, à exceção de PUVA e methotrexate. Menos de 40\% dos usuários indicaram que estiveram satisfeitos com alguma das quatro terapias avaliadas. Para a maior parte nenhuma das quatro terapias, utilizadas extensamente em 2002, para o tratamento de psoríase, era altamente satisfatória.

Arruda, Campbell eTakahashi (2001) acrescentam que a escolha do tratamento deve ser feita considerando-se a gravidade, a extensão do quadro clínico e o comprometimento emocional. No tratamento devem ser priorizadas, inicialmente, medidas gerais e terapia tópica, a seguir fototerapia e, por último, tratamento sistêmico.

Lipp (1991), Dias et al. (1996) e Dias e Lipp (2001) ressaltam a importância do controle de stress e técnicas cognitivas no tratamento adjuvante da psoríase. As técnicas cognitivas e comportamentais contribuem para uma adaptação à doença, ajudam a desenvolver estratégias psicológicas de enfrentamento e colaboram

260 para a melhora na qualidade de vida.
Considerando-se que a psoríase tem uma relação muito íntima com a área emocional, no seu fator desencadeador ou potencializador, Steiner e Perfeito (2003) mencionam que o stress físico ou emocional tem repercussões em inúmeras dermatoses. Essas, indiscutivelmente, também são geradoras de stress. Para o dermatologista, é fundamental ponderar como significativa essa relação, que ocorre em grande número de doenças de pele, além de sempre procurar avaliar a integração entre sentimentos, conflitos e stress em cada paciente.

Evidências práticas e científicas comprovam que a vida moderna apresenta muitos recursos, equipamentos e benefícios que ajudam as pessoas, mas é acompanhada de agentes estressantes que, muitas vezes, quando não administrados, podem prejudicar o bem-estar e a saúde da população. Nesse contexto, a psicoterapia cognitivo-comportamental pode ajudar o paciente com psoríase a enfrentar melhor o quadro clínico. Entretanto, para que se possa lidar especificamente com o stress, inicialmente, deve-se saber o que vem a ser essa reação do organismo.

\section{Stress}

Conforme Atkinson, Atkinson, Smith, Bem e Hoeksema (1995), stress é um estado que ocorre quando a pessoa se depara com eventos que colocam em perigo seu bem-estar físico ou psicológico. As tentativas para a adaptação à presença contínua de um fator estressor podem esgotar os recursos do corpo e torná-lo mais vulnerável à doença.

Torrezan (1997) concorda que o stress excessivo promove desgaste do organismo e aumenta a suscetibilidade às doenças, tendo relação com seu aparecimento ou agravamento.

De acordo com Lipp (2003), o stress é uma reação com componentes físicos, químicos, mentais e emocionais do organismo, causada pelas alterações psicofísiológicas que ocorrem quando a pessoa se confronta com uma situação que possa irritá-la, amedrontá-la, confundi-la ou fazê-la feliz. Fundamentando-se nos estudos de Selye (1965) sobre o assunto, Lipp (2000) refere que a reação do stress pode apresentar níveis diferentes de intensidade e de características e estabelece uma classificação do stress com base em quatro fases: 
a) Fase de alerta: caracteriza o momento do contato com a situação estressora, com manifestações somáticas e psíquicas, tais como taquicardia, suor e tensão muscular.

b) Fase de resistência: quando o estressor é de longa duração, ou sua intensidade é em demasia para a resistência da pessoa, o organismo tenta reequilibrar a homeostase interna e para isso utiliza sua energia adaptativa. Alguns sintomas podem ocorrer, como dificuldades com a memória, irritabilidade e cansaço excessivo.

c) Fase de quase-exaustão: manifestada quando as defesas do organismo começam a ceder e ele já não consegue resistir às tensões ou restabelecer o equilíbrio interior. É comum a pessoa sentir que oscila entre momentos de bem-estar e tranqüilidade e momentos de desconforto, cansaço e ansiedade. As doenças podem aparecer nessa etapa ou, caso a pessoa já esteja doente, pode haver um agravamento do quadro.

d) Fase de exaustão: caracteriza-se pela permanência dos agentes estressores de forma constante e intensa, com agravamento dos sintomas físicos e psicológicos e pela impossibilidade de lidar de forma efetiva com tal situação. Alguns sintomas mais comuns dessa fase podem ser: perda do senso de humor, alteração do apetite, apatia, depressão, raiva e desgaste físico.

A reação do stress ocorre quando algo ameaça ou prejudica a vida de um indivíduo. Esse fator ameaçador é também denominado de estressor, agente, fonte, estímulo e causador do stress. O que caracteriza um evento como estressor é sua capacidade de romper o equilíbrio do organismo, levando-o a um conjunto de reações na busca de adaptação à situação imposta.

Segundo Atkinson et al. (1995), incontáveis eventos ajudam no aparecimento de stress; alguns deles são determinados por fatores externos, mudanças importantes que afetam as pessoas, tais como: guerra, acidentes nucleares, terremotos, mudança de cidade, de emprego, casamento, perda de um ente querido e doença grave.

A fonte de stress também pode estar dentro do indivíduo, na forma de motivos ou desejos conflitantes. O stress causado por processos internos, conflitos não resolvidos, podem ser conscientes ou inconscientes: quando o indivíduo se depara com uma escolha entre objetivos incompatíveis ou exclusivos, ou quando tem de optar entre dois objetivos igualmente atraentes, ou ainda quando duas necessidades ou motivos íntimos estão em oposição um com o outro (conflitos internos entre objetivos rivalizáveis).

Os eventos percebidos como estressantes ajustam-se nas seguintes categorias: eventos traumáticos (fora da faixa habitual de experiências humanas), eventos incontroláveis, imprevisíveis e eventos que desafiam os limites de capacidades e autoconceito, ou conflitos internos.

O stress crônico pode estar correlacionado a transtornos físicos, tais como: úlcera, hipertensão arterial, doenças do sistema gástrico, respiratório ou dermatológico. A reação do stress também pode prejudicar o sistema imunológico, diminuindo a capacidade do organismo para combater bactérias e vírus invasores (Rodrigues, 1997). Segundo Lipp e Guevara (1994), o stress é considerado um elo tão intenso entre mente e corpo que nenhuma doença ou qualquer outra condição produz tal interação.

Vive-se, atualmente, em um mundo no qual as fontes de stress são inúmeras - desemprego, dificuldades financeiras, insegurança, dentre outras -, causando impacto sobre o indivíduo. Lipp (2003) comenta que a maior parte do dia-a-dia das pessoas gera algum stress. As situações estressantes variam e são diferentes para cada um. A maioria das pessoas sofre com o stress e, de alguma maneira, demonstra tal sofrimento.

Ainda segundo Lipp (1991), o stress, seja de características predominantemente psicológicas ou físicas, vem como conseqüência da dificuldade em lidar com os sentimentos e conseqüentemente a pele passa a funcionar como fonte de expressão, podendo manifestar essa relação por meio das dermatoses decorrentes da sintomatologia do próprio stress. Nesse sentido, dentre os possíveis fatores associados ao estabelecimento da evolução da psoríase destaca-se o stress.

\section{Psoríase, aspectos psicológicos e stress}

Para diversos estudiosos, a pele, além de outras funções, também representa a pessoa como indivíduo único. De acordo com Chiozza (1991), nota-se que portadores de psoríase, mais que outros com doenças de pele, sentem-se desprezíveis, sujos e intocáveis. Temem ser isolados, rejeitados e apresentam fantasias de abandono. Sentem a exclusão como falta de reconhecimento, no sentido da aceitação de sua identidade, 
como a rejeição que os coloca em uma classe, casta ou condição inferior; sentem-se possuidores de uma identidade repugnante e sofrem frente a uma sociedade que estabelece padrões ideais de beleza e de adequação. O problema da pele acaba favorecendo sensações de discriminação, inadequação e insatisfação quanto à aparência física.

Situações desse tipo, acrescidas da própria constituição da personalidade, fundamentada em uma imagem corporal debilitada pela doença, podem também influenciar na capacidade de auto-aceitação e satisfação pessoal do indivíduo.

De acordo com Gon, Rocha e Gon (2005), a dermatite atópica, a psoríase e o vitiligo são doenças de pele crônicas em que o prognóstico, a evolução, os tipos de cuidados requeridos e a visibilidade da condição constituem fatores que afetam o comportamento do paciente e de seus familiares. A grande dificuldade enfrentada pelo portador de uma dessas doenças é se tornar alvo constante de discriminação e de preconceito.

Gon et al. (2005), verificaram alguns efeitos de comportamentos estigmatizantes sobre o relato verbal de crianças portadoras de dermatoses crônicas, a partir de personagens de uma história infantil. Participaram da pesquisa seis crianças entre sete e oito anos de idade; elas criaram, individualmente, um texto para as personagens da história. Os resultados mostraram que o preconceito e a estigmatização estão presentes em diferentes contextos sociais e que existem dificuldades tanto das crianças quanto da família para enfrentarem de modo adequado essas situações.

Conforme Goffman (1988), a sociedade cria um modelo de normalidade imposto a todos. Aquele que, por alguma razão, não se encaixar nesse modelo é visto como um desigual e não apenas como diferente. 0 estigma despoja a pessoa de sua identidade social, esvaindo-se de seus atributos de humanidade.

Pesquisas demonstram que, no caso da psoríase, os sentimentos de rejeição e de estigmatização podem estar presentes no cotidiano do paciente, podendo comprometer sua adaptação social, com os conseqüentes problemas no ambiente de trabalho, dificuldades em situações públicas, como cabeleireiros, clubes, mudança na forma de se vestir, além de significativas sensações de irritação e de angústia. Devido à

262 aparência física da doença e à auto-imagem preju- dicada, o indivíduo pode ficar mais vulnerável ao stress (Silva, 2003).

Lipp (2003) salienta que, por um lado, a psoríase está relacionada à conseqüência do stress, pois muitos pacientes referem desencadeamento ou piora das lesões a partir de situações estressantes. Por outro lado, a psoríase está freqüentemente vinculada ao desencadeamento de stress emocional pelo constrangimento em relação às próprias lesões da doença. A percepção dos eventos estressantes seria acompanhada pela subjetividade de cada um, caracterizando a vivência como mais ou menos estressora, o que provavelmente pode contribuir para a piora ou melhora da doença, em termos de expectativa.

Outro fator a ser destacado é a habilidade do indivíduo em lidar com a doença, ou seja, a capacidade adaptativa (coping) para enfrentar e desenvolver estratégias que possibilitem administrar os sintomas da psoríase (Silva, 2003).

Pesquisas na área relacionam psoríase, aspectos psicológicos e stress, como o estudo realizado por Lipp (1991) com pessoas portadoras dessa doença. O trabalho objetivou avaliar o resultado do tratamento de controle do stress emocional com a redução e a recidiva das lesões no seu tamanho e extensão. Participaram dessa pesquisa 20 adultos, de 19 anos a 60 anos, com diagnóstico de psoríase. Os pacientes foram divididos em dois grupos: experimental, que recebeu tratamento médico e treino de controle do stress, e o grupo-controle, que recebeu somente tratamento médico e avaliações psicológicas. Concluiu-se que os fatores emocionais e o alto grau de stress foram responsáveis pelo agravamento e/ou reaparecimento da psoríase. Todos os pacientes revelaram ser sensíveis, preocupados, inconformados, ansiosos, nervosos e irritados.

Estudo realizado por Mazzetti et al. (1994) sobre psoríase, stress e desordens psiquiátricas, objetivou enten-der como os eventos estressores podem determinar a recaída da psoríase. A pesquisa usou avaliações clínicas com os testes de Rorschach, MMPI (Inventário Multifásico da Personalidade da Universidade de Minnesota) e HTP (Desenho da Casa, Árvore, Pessoa), em 80 pacientes. Observou-se que 71,20\% dos participantes apresentaram desordens psiquiátricas, sendo 35,00\% com transtornos da personalidade; $17,50 \%$ tristeza e melancolia; $12,50 \%$ ansiedade e 6,25\% esquizofrenia. Verificou-se, em 88,70\% 
dos pacientes, a presença de eventos estressantes. Os autores sugerem que a piora do quadro da psoríase esteja ligada à interpretação da experiência demonstrada pela pessoa diante do evento ou situação estressante.

Pesquisa de Dias et al. (1996) objetivou verificar se o controle da assertividade é útil no tratamento coadjuvante da psoríase. Participaram do estudo dez pacientes com a doença, avaliados quanto ao stress, assertividade, crenças irracionais e qualidade de vida. Foram consultados pelos médicos quanto à extensão das placas psoriáticas e a percentagem corpórea afetada. As avaliações psicológicas e médicas foram comparadas nas fases pré e pós-tratamento.

Os resultados mostraram que, após o tratamento psicológico de cinco sessões, quatro pacientes apresentaram uma diminuição, quatro outros permaneceram inalterados e dois aumentaram a percentagem de área afetada pela psoríase. Quanto à avaliação psicológica, não houve melhora na manifestação de sintomas do stress. Entretanto, em relação à assertividade, oito pacientes apresentaram uma diminuição do número de respostas inassertivas; desses, quatro mostraram uma diminuição significativa. Sugerem os autores que a redução da área afetada pode não ter sido espontânea e sim devido ao treino de assertividade.

Estudo sobre a relação entre o stress e a psoríase foi realizado por Dias e Lipp (2001), que avaliaram 14 pacientes com psoríase (sendo sete sujeitos por grupo), com idade de 23 a 70 anos, predominantemente mulheres. O grupo comparativo I (GCl) recebeu o treino de controle de stress (TCS) com treino de assertividade e crenças irracionais, e o grupo comparativo II (GCII) recebeu apenas o treino de assertividade no TCS. Os resultados demonstraram diferenças estatisticamente significantes. O grupo GCl mostrou melhoras na extensão das lesões afetadas pela psoríase, quando trabalhadas as dimensões inassertividade e crenças irracionais. O grupo GC II não apresentou melhoras significativas quanto às lesões da psoríase. Esses dados demonstraram a importância da reestruturação cognitiva no TCS para pacientes com psoríase.

Estudo realizado por Silva (2003), com oito pacientes, ambos os sexos, com média de 33 anos de idade, diagnóstico de psoríase confirmado, teve como objetivos avaliar o nível de stress e verificar a relação entre eventos estressantes e o aparecimento e/ou agravamento da doença em portadores de psoríase. Os instrumentos utilizados para a pesquisa foram: entrevista semi-estruturada e Inventário de Stress para Adultos de Lipp (2000). Os dados mostraram que 75,0\% dos participantes não apresentavam sintomas significativos do stress. Contudo aqueles que apresentavam stress se encontravam na fase de estabilidade da doença; $62,5 \%$ dos entrevistados relacionavam eventos ou acontecimentos na vida com o aparecimento da doença e 87,5\% associavam eventos ou acontecimentos na vida com a melhora ou piora do quadro clínico da psoríase.

Metade da amostra possuía algum parente na família com diagnóstico da doença; e em 25,0\% dos participantes, a psoríase comprometeu a aparência física. Para relacionamentos interpessoais, 62,5\% relataram não comprometimento, e para relacionamento familiar, 75,0\% não apresentavam dificuldades. A maior parte dos entrevistados relatou que possuía amigos com quem se relacionava. A amostra não se encontrava estressada e estava na fase de estabilidade da doença; esse dado pode sugerir que essas pessoas possuíam estratégias de controle de stress (diário), atuando de modo profilático para o controle da psoríase. Os resultados também sugerem que as alterações emocionais estavam relacionadas com a melhora ou piora da doença.

Alguns estudiosos investigaram a relação dos fatores emocionais, inclusive o stress e o aparecimento ou a piora da psoríase. Zachariae et al. (2004) pesquisaram a influência do stress para o aparecimento e agravamento da psoríase, comparando stress e outras reações associadas à doença, bem como a gravidade, o histórico familiar e fatores sociodemográficos. Participaram da amostra um total de 5.795 integrantes de uma associação de portadores de psoríase e 702 pacientes recrutados pelos dermatologistas e pelas clínicas universitárias nórdicas.

Investigou-se, com os participantes, se o aparecimento da psoríase ocorreu durante épocas de preocupação e de stress. Avaliaram-se o grau em que a psoríase foi influenciada pelo stress, o índice do stress na qualidade de vida, o índice da inabilidade da psoríase e fatores sociodemográficos. Os resultados mostraram que 71\% dos integrantes da associação e 66\% dos pacientes informaram que a psoríase era exacerbada com o stress e 35\% nos dois grupos informaram que o início da psoríase ocorreu durante o período de preocupação e stress.

Os participantes mencionaram que o aumento da gravidade da doença estava ligado ao stress e a 
melhora da doença estava relacionada a uma qualidade de vida melhor. Também informaram que faziam uso freqüente de tabaco, tranqüilizantes e antidepressivos. Mais mulheres do que homens apresentaram reações de stress. Assim os autores concluíram que a pesquisa confirma e estende resultados de estudos precedentes e indica que um subgrupo de pacientes com psoríase pode ter reações psicológicas associadas à doença que influenciam sua vida diária.

Richards, Fortune, Griffiths e Main (2001) avaliaram a importância geral e específica de aspectos psicológicos em pacientes com psoríase; examinaram o quadro clínico da doença e as variáveis psicológicas a ela relacionadas. Foram investigados 115 pacientes, que responderam a um questionário psicológico e a outro específico sobre psoríase. Verificaram-se altos níveis de stress na amostra; $43 \%$ dos pacientes com tendência à ansiedade e 10\% com tendências à depressão. A gravidade do quadro clínico da psoríase e a área afetada não tiveram nenhum impacto no stress excessivo psicológico e na inabilidade psicológica. As percepções da estigmatização foram relacionadas significativamente ao stress excessivo psicológico e ao grau de inabilidade. Concluíram que fatores psicológicos foram os determinantes mais significativos da incapacidade dos pacientes com psoríase do que a gravidade, localização ou duração da doença. Esses fatores têm importante implicação na relação com a administração do quadro clínico da psoríase.

Pesquisa realizada por Vardy, Amir, Gesthalter, Biton e Buskila (2002) objetivou avaliar níveis de estigmatização em dois grupos de pacientes portadores de doenças dermatológicas. Participaram do estudo 100 pacientes com psoríase e 100 pacientes com diversas doenças dermatológicas. Os resultados mostraram que os pacientes com psoríase experienciaram níveis mais elevados de estigmatização quando comparados com pacientes portadores de outros problemas de pele.

Russo, Ilchef e Cooper (2004), em um estudo bibliográfico, constataram que a psoríase é uma condição comum, afetando de 1,5 a 2,0\% da população dos países industrializados. Os autores ressaltam que a doença tem impacto emocional no indivíduo portador, não relacionado necessariamente à extensão da doença da pele. Na pesquisa de levantamento de dados, os autores referiram que a psoríase estava associada com 264 uma variedade de problemas psicológicos, incluindo baixa auto-estima, disfunção sexual, depressão e ideação suicida. Acrescentaram a importância de os clínicos considerarem os aspectos psicológicos que envolvem essa doença.

Marot (1993) refere que em uma amostra de pacientes dermatológicos, utilizando o Structured Clinical Interview of Diagnóstic (SCID) para estudar a freqüência dos transtornos de ansiedade e depressão nessa população, observou que $35,7 \%$ dos pacientes apresentaram pelo menos ansiedade ou depressão. $\mathrm{O}$ autor ressalta que devido a essa alta freqüência, é necessário tomar precauções para diminuir o sofrimento desses pacientes e seu tempo de internação.

Estudo sobre fatores econômicos, sociais e profissionais em pacientes com psoríase foi realizado por Pellerano et al. (1997). Os autores referem que a psoríase tem um forte impacto sobre a qualidade de vida dos pacientes, principalmente na esfera psicossocial, afetando aspectos pessoais, profissionais, da área sexual e suas relações afetivas.

Um questionário com 43 perguntas, aplicado a 103 pacientes no ano de 1996, objetivou registrar se os pacientes tinham conhecimento de sua doença; quais os tratamentos prévios efetuados e o impacto socioeconômico da psoríase em suas vidas, bem como as coincidências e as discrepâncias relativas aos resultados de um questionário similar, realizado em 1973. No atual estudo, concluíram que a psoríase teve um impacto sobre a qualidade de vida, constataram a falta de informação e de orientação que apresentaram os pacientes ao longo dos anos, além da influência negativa na comunicação com os médicos.

Mingorance, Loureiro, Okino e Foss (2001) tiveram como objetivo avaliar as condições psicológicas e adaptativas de pacientes com psoríase e suas relações com variáveis demográficas e condições clínicas. No Ambulatório de Dermatologia do Hospital das Clínicas da Faculdade de Medicina de Ribeirão Preto da Universidade de São Paulo, foram investigados 25 mulheres e 25 homens na faixa etária entre 20 e 50 anos, com diagnóstico de psoríase vulgar. Procedeu-se ao uso do Inventário Simplificado de Personalidade (ISP) e do Inventário de Qualidade de Vida (PDI). Os dados indicaram que $80 \%$ dos pacientes apresentaram tendência a desvio de traço de personalidade, predominando as escalas de Extroversão, Insanidade e Neuroticidade. 
A maior parte relatou dificuldades de adaptação psicossocial, principalmente nas áreas de atividades rotineiras ( $p<0,001)$; extensão da lesão da área afetada pela psoríase e insatisfação quanto à aparência física. Desvios de insanidade e neuroticidade mostraram-se associados a prejuízos nas atividades rotineiras, nas relações pessoais e no lazer. Os dados apontam a inter-relação de aspectos psíquicos e adaptação psicossocial com peculiaridades clínicas da doença.

Rodríguez Vivas e Sosa Sarritiello (2002), realizando entrevistas clínicas em uma amostra com 76 pacientes de ambulatório, observaram uma alta prevalência de transtornos de ansiedade e, menos freqüentemente, transtornos de personalidade e de humor; foram identificadas dificuldades associadas à imagem corporal e às relações com outras pessoas, e alguns aspectos foram relevantes sobre a sexualidade dos portadores de psoríase.

Gupta e Gupta (2003) comentam sobre a importância dos fatores psiquiátricos e psicológicos em pelo menos 30\% dos problemas dermatológicos. Em muitos casos, foi observado o impacto das lesões da pele na qualidade de vida. Em determinados problemas, como a acne e a psoríase, a comorbidade psiquiátrica pode ser associada com as emergências psiquiátricas, como exemplo, o suicídio.

\section{Considerações Finais}

A consideração de fatores psiquiátricos e psicológicos é importante para avaliação e tratamento e para alguns aspectos da prevenção de problemas dermatológicos. O modelo biopsicossocial permite fazer uma avaliação psicológica e verificar o fator social, além dos fatores dermatológicos preliminares.

Os dados da literatura na área mostram a estreita relação entre os aspectos psicológicos, emocionais e o stress no aparecimento e agravamento da psoríase. Ressaltam que o clínico deve olhar para o paciente e considerar seu estado emocional, assim como sugerir tratamento psicoterápico a fim de colaborar para a melhora do quadro clínico, bem-estar e qualidade de vida dos portadores dessa doença estigmatizante.

Este artigo teve por objetivos informar e comentar alguns dos estudos sobre psoríase, aspectos psicológicos, stress e eventos de vida, bem como apresentar estratégias de intervenção e incentivar o interesse por novas explorações na área.

Os autores citados concordam quanto à possibilidade de o fator genético ser uma predisposição para a manifestação da doença, pois a maior parte dos pacientes com psoríase apresenta casos na família. Concordam também que a implicação emocional da doença afeta a imagem, o que pode levar à estigmatização social. Observa-se a congruência quanto à posição de que o estado emocional e o stress podem provocar o aparecimento e a piora das doenças dermatológicas.

Esta consulta à literatura pode auxiliar profissionais que atuam com pacientes portadores da doença a identificarem e tratarem com auxílio da psicoterapia cognitivo-comportamental, além de propor tratamento médico para uma melhora da qualidade de vida dos pacientes.

Os profissionais da área de saúde devem considerar o indivíduo como um ser biopsicossocial e levar em conta características individuais, físicas, psicológicas, familiares, meio social e estilo de vida. O estudo dessas características individuais pode colaborar para a adaptação, profilaxia, tratamento e maneira de gerenciar os fatores desencadeadores e/ ou potencializadores da doença.

\section{Referências}

Allegranti, I., Gon, T., Magaton-Rizzi, G., \& Aguglia, E. (1994). Prevalence of alexithymic characteristics in psoriatic patients. Acta Dermato Venereologica Supplementum (Stockh), 186, 146-147.

Arruda, L. H. F., Campbell, G. A. M., \& Takahashi, M. D. F. (2001). Psoríase. Anais Brasileiros de Dermatologia, 76 (2), 141-167.

Atkinson, R. L., Atkinson, R. C., Smith, E. E., Bem, D. J., \& Hoeksema S. N. (1995). Estresse e enfrentamento. In R. L. Atkinson, R. C. Atkinson, E. E. Smith, D. J. Bem \& S. N. Hoeksema. Introdução à Psicologia (11a. ed., pp.458-483). Porto Alegre: Artes Médicas.

Berkow R., Beers M. H., Bogin R. M., \& Fletcher A. J. (2003). Manual Merck de informação médica: saúde para a família (pp.1033-1034). São Paulo: Manole.

Bork, K., \& Brauninger, W. (1998). Dermatologia clínica: diagnóstico e terapia (2a. ed., pp.127-130). São Paulo: Manole.

Brophy, S., Taylor, G., Blake, D., \& Calin, A. (2003). The interrelationship between sex, susceptibility factors, and outcome in ankylosing spondylitis and its associated disorders including inflammatory bowel diseases, psoriasis, and irits. Jounal Rheumatol, 30 (9), 2054-2058. 
Chiozza, L. A. (1991). Os afetos ocultos em psoríase, asma, transtornos respiratórios, varizes, diabetes, transtornos ósseos, cefaléias e acidentes celebrovasculares (pp.19-42). São Paulo: Casa do Psicólogo.

Dias, R. R., Barbosa, A. P., Moura, B. S. C., Aguiar, C. M. O. M., Leite, C. R., Gallo, C. E., Aidar, I., Carvalho, L. A. S. B., Gomes, M. A., Ferreira, R. M., Laloni, D. T., \& Lipp, M. N. (1996). Investigação da relação entre stress e psoríase. Anais do 10 Simpósio sobre Stress e suas implicações: um encontro internacional (p.111). Campinas.

Dias, R. R., \& Lipp, M. N. (2001). Estresse e doença crônica. Resumos-X Encontro Brasileiro de Psicoterapia e Medicina Comportamental (pp.59-60). Campinas, São Paulo.

Elder, D., Elenitsas, R., loffreda, M., Miller, J., Miller, O. F., \& Johnson, B. (2001). Histopatologia da pele de lever: manual e atlas (pp.93-100). São Paulo: Monole.

Goffman, E. (1988). Estigma (pp.11-41). Rio de Janeiro: Guanabara.

Gon, M. C. C., Rocha, M. M., \& Gon, A. S. (2005). Análise do conceito de estigma em crianças com dermatoses crônicas. Revista Brasileira de Terapia Comportamental Cognitiva, 7 (1), 15-20.

Gupta, M.A., \& Gupta, A.K. (2003). Psychiatric and psychological co-morbidity in patients with dermatologic disorders: epidemiology and management. American Journal Clinical Dermatology, 4 (12), 833-42.

Lipp, M. E. N. (1991). Estudos experimentais de duas condições de tratamento médico-psicológico a pessoa portadora de psoríase. Revista do Núcleo de Estudos Psicológicos. Campinas: Unicamp.

Lipp, M. E. N., \& Guevara, A. J. H. (1994). Validação empírica do inventário de sintomas de stress. Estudos de Psicologia, 11 (3), 42-49.

Lipp, M. E. N. (2000). Inventário de sintomas de stress para adultos de Lipp. São Paulo: Casa do Psicólogo.

Lipp, M. E. N. (2003). O modelo quadrifásico do stress. In M. E. N. Lipp (Org), Mecanismos neuropsicofisiológicos do stress: teoria e aplicação clínica (pp.17-22). São Paulo: Casa do Psicólogo.

Marot, R. S. V. (1993). Distúrbios de ansiedade e depressão na dermatologia. Anais Brasileiros de Dermatologia, 68 (5), 251-252.

Mazzetti, M., Mozzetta, A., Soavi, G. C., Andreoli, E., Foglio Bonda, P. G., Puddu, P., \& Decaminada, F. (1994). Psoriasis, stress and psychiatry: psychodynamic characteristics of stressors. Acta Dermato Venerreologica Supplementum (Stockh), 186, 62-4.

McDougall, J. (1996). Teatros do corpo: o psicossoma em psicanálise (2a. ed., pp.33-52). Rio de Janeiro: Tempo Brasileiro.

Mingorance, R. C. (1999). Pacientes com psoríase: características de personalidade, nivel de stress e adaptação psicossocial. Dissertação de mestrado não-publicada, Faculdade de Filosofia, Ciências e Letras de Ribeirão Preto, Universidade de São Paulo, Ribeirão Preto.

Mingorance, R. C., Loureiro, S. R., Okino, L., \& Foss, N. (2001). Pacientes com psoríase: adaptação psicossocial e características de personalidade. Medicina (Ribeirão Preto), 34 (3/4), 315-324.

Nijsten, T., Margolis, D. J. K., Feldaman, S. R., Rolstad, T., \& Stern, R. S. (2005). Traditional systemic treatmentshave not fully met the needs of psorisis patients: results from a national survey. Journal of American Academy of Dermatology, 52 (3), 434-44.

Pellerano, G., Neglia, V., Harris, P., Poggio, N., León, S., De Sena, A., \& Chouela, E. (1997). Aspectos económicos, sociales y laborales de la psoriasis. Archivos Argentinos de Dermatologia, 47 (6), 263-268.

Proença, N. G., \& Maia, M. (1995). Psoríase. In O. L. Ramos \&H. A. Rothschild. Atualização terapêutica: manual prático de diagnóstico e tratamento (17a. ed., pp.606-6070). Porto Alegre: Artes Médicas.

Richards, H. L., Fortune, D. G., Griffiths, C. E., \& Main, C. J. (2001). The contribuition of perceptions of stigmatization to disability in patients with psoriasis. Journal of Psychosomatic Research, 50 (1), 11-15.

Rodrigues, R. S. (1997). Contribuições de técnicas de exame psicológico com crianças asmáticas efamiliares. Dissertação de mestrado não-publicada, Faculdade de Medicina de Ribeirão Preto, Universidade de São Paulo, Ribeirão Preto.

Rodríguez Vivas, C. J., \& Sosa Sarritiello, L. (2002). Cinco casos demostrativos de un estudio psicomástico en enfermos psoriáticos. Archivos Del Hospital Vargas, 44 (1/2), 60-67.

Russo P. A., Ilchef R., \& Cooper, A. J. (2004). Psychiatric morbidity in psoriasis: a review. Australasian Journal of Dermatology, 45 (3), 155-159.

Sampaio, S. A. P., Castro, R. M., \& Rivitti, E. A. (1989). Dermatologia básica. São Paulo: Artes Médicas.

Selye, H. (1965). Stress: a tensão da vida (2a. ed.). São Paulo: Ibrasa.

Silva, K. (2003). Stress em pacientes com psoríase. Trabalho de conclusão de curso não-publicado, Universidade Camilo Castelo Branco, São Paulo.

Steiner, D., \& Perfeito, F. L. (2003). A relação entre stress e doenças dermatológicas. In M. E. N. Lipp, (Org.), Mecanismos neuropsicofisiológicos do stress: teoria e aplicação clínica (pp.111-114). São Paulo: Casa do Psicólogo.

Torrezan, E. A. (1997). As implicações do stress nas doenças. Revista Psico-USF, 2 (1), 13-22.

Vardy, D., Besser, A., Amir, M., Gesthalter, B., Biton, A., \& Buskila, D. (2002). Experiences of stigmatization play a role in mediating the impact of disease severity on quality of life psoriasis patients. The British Journal of Dermatology, 147 (4), 736-742.

Zachariae R., Zachariae, H., Blomqvist, K., Davidsson, S., Molin, L., Mork, C., \& Sigurgeirsson, B. (2004). Self-reported stress reactivity and psoriasis-related stress of Nordic psoriasis sufferers. Journal European Academy of Dermatology and Venereology, 18 (1), 27-36.

Recebido em: 30/6/2005

Versão final reapresentada em: 28/10/2006

Aprovado em: 3/11/2006 